\title{
Animal models of SARS-CoV-2 infection and mechanisms of COVID-19
}

\author{
Simon Cleary ${ }^{1}$, Simon Pitchford ${ }^{2}$, Richard Amison ${ }^{2}$, Robert Carrington ${ }^{3}$, C.Lorena \\ Robaina Cabrera ${ }^{2}$, Melia Magnen ${ }^{4}$, Mark Looney ${ }^{4}$, Elaine Gray $^{5}$, and Clive Page ${ }^{2}$ \\ ${ }^{1} \mathrm{UCSF}$ \\ ${ }^{2}$ King's College London \\ ${ }^{3}$ Covance Laboratories Ltd \\ ${ }^{4}$ University of California San Francisco \\ ${ }^{5}$ National Institute for Biological Standards and Control
}

May 4, 2020

\begin{abstract}
The coronavirus disease 2019 (COVID-19) pandemic caused by SARS-CoV-2 infections has led to substantial unmet need for treatments, many of which will require testing in appropriate animal models of this disease. Vaccine trials are already underway, but there remains an urgent need to find other therapeutic approaches to either target SARS-CoV-2 or the complications arising from viral infection, particularly the dysregulated immune response and systemic complications which have been associated with progression to severe COVID-19. At the time of writing, in vivo studies of SARS-CoV-2 infection have been described using macaques, cats, ferrets, hamsters, and transgenic mice expressing human angiotensin I converting enzyme 2 (ACE2). These infection models have already been useful for studies of transmission and immunity, but to date only partially model the mechanisms implicated in human severe COVID-19. There is therefore an urgent need for development of animal models for improved evaluation of efficacy of drugs identified as having potential in the treatment of severe COVID-19. These models need to recapitulate key mechanisms of COVID-19 severe acute respiratory distress syndrome and reproduce the immunopathology and systemic sequelae associated with this disease. Here, we review the current models of SARS-CoV-2 infection and COVID19-related disease mechanisms and suggest ways in which animal models can be adapted to increase their usefulness in research into COVID-19 pathogenesis and for assessing potential treatments.
\end{abstract}

\section{Hosted file}

Cleary SJ et al 2020 .pdf available at https://authorea.com/users/317432/articles/447533-animalmodels-of-sars-cov-2-infection-and-mechanisms-of-covid-19 